\title{
Mechanisms underlying the association between breastfeeding and obesity
}

\author{
CYNTHIA J. BARTOK ${ }^{1} \&$ ALISON K. VENTURA ${ }^{2}$ \\ ${ }^{1}$ Department of Kinesiology, The Pennsylvania State University, University Park, PA, ${ }^{2}$ Monell Chemical Senses Center, \\ Philadelphia, PA
}

\begin{abstract}
Decades of epidemiological research have established that breastfeeding is associated with a modest reduction in risk of later overweight and obesity. However, no systematic effort has been made to delineate the mechanisms that may explain this association. This review summarizes evidence from a variety of disciplines to understand the potential mechanisms underlying this association. One possibility is that this association is spurious and that confounding factors fully or partially explain this association. Additionally, breastfeeding could confer protection by: encouraging the infant's emerging capabilities of self-regulation of intake; reducing problematic feeding behaviors on the part of caregivers that interfere with the infant's self-regulation of intake; and providing bioactive factors that regulate energy intake, energy expenditure, and cellular chemistry. These three protective effects may promote slower growth and lower body fat levels in breastfed infants, which reduce risk of overweight and obesity later in life.
\end{abstract}

Key words: Breastfeeding, bottle-feeding, infant formula, growth, child development, parenting, overweight

Despite improvements in the health of the world's children and adults during recent decades, the obesity epidemic now presents a major public health challenge for the coming decades (1). Rates of overweight and obesity are rising in nearly all countries around the globe, and even infants and preschoolers are affected by this public health crisis (2). Because treatment of childhood obesity has shown limited success $(3,4)$, research efforts have become focused on prevention (5).

Recent public health and research initiatives have identified the first years of life as a critical period for targeting the prevention of later obesity $(4,6,7)$. One highly researched area within this field is the study of what (breast milk, formula) and how (breast, bottle) infants are fed and how this relates to later obesity risk (5). Kramer (8) was the first to report an association between breastfeeding and reduced risk of overweight and obesity in 12 to 18 -year-olds. Subsequent studies have largely confirmed that this statistical association is seen in children as young as 3 years of age (9), during adolescence (10), and in adulthood (11). These studies have suggested that both exclusivity and duration of breastfeeding strengthen the association $(10,12,13)$. The consensus based on reviews and meta-analyses is that breastfeeding provides modest protection against later overweight, and that increasing exclusivity and duration strengthens this protective effect (14-17).

As these conclusions are based on epidemiological studies describing associations between breastfeeding and later overweight and obesity, a clear limitation of the field is the inability to draw conclusions about causation. Research has demonstrated that the probability of success with public health interventions is greater when the evidence base supports a causal or mechanistic relationship $(18,19)$. Thus, the purpose of this review is to compile evidence from a variety of disciplines to understand the potential mechanisms that may explain the association between breastfeeding and decreased risk for overweight and obesity. In addition, this review identifies several modifiable aspects of infant feeding 
practices that could be targeted and promoted in intervention and prevention studies.

\section{Potential explanations for the association}

This review will address three possible explanations for the association between breastfeeding and later weight status. One explanation is that confounding factors, which could be the "true" cause of the protective effect, create a spurious effect between breastfeeding and later weight status. One alternative explanation is that behavioral differences in motherinfant dyads that stem from either breastfeeding or bottle-feeding produce different outcomes. Another alternative explanation is that the differences in the composition and/or constituents of breast milk and formula produce different outcomes. Research from a wide disciplinary base suggests that both how and what an infant is fed may affect short- and long-term risk for overweight.

For the purposes of this review behavioral and physiological factors will be discussed separately for clarity and parsimony. However, the combined interactions and effects of these components are inseparable and likely to have an interactive, dynamic effect on the infant's feeding experience. As the review subsequently discusses, this net feeding experience produces differences in infant growth, weight status, and adiposity through its intermediary effect on the metabolic profile of the body (metabolome) and the net balance of energy intake versus expenditure (energy balance) (20). The remainder of this review will examine the evidence base for these potential explanations underlying the association between breastfeeding and later weight status.

\section{Confounding factors}

One possible explanation for the association between breastfeeding and reduced risk of obesity is confounding factors. Confounding factors, such as maternal weight status, education, socioeconomic status, and age, indirectly influence child growth and weight status independently of the infant feeding experience. Thus, the association between breastfeeding and later obesity in self-selected feeding situations may in part or wholly be due to other confounding lifestyle factors, rather than the infant feeding experience per se.

In industrialized countries, mothers who choose to breastfeed and who breastfeed longer are typically more educated, wealthier, older and have more social support for breastfeeding (21). These same maternal factors are statistically associated with healthier lifestyle practices, such as regular physical activity and healthier diets $(22,23)$. In addition, overweight mothers are statistically more likely to have overweight children (24) but are also statistically less likely to initiate and continue breastfeeding $(25,26)$.

Based on these confounding factors, some researchers have concluded that lifestyle factors explain the association between infant feeding experience and overweight risk (27). To tease out the effects of confounding factors, cohort studies typically attempt to statistically control for known confounding factors (e.g., [16]), which reduces but does not eliminate the association between breastfeeding and obesity. However, this approach controls only for the known confounding factors and may not fully control for the complex effects of lifestyle factors, such as physical activity. Other investigators have studied sibling pairs, who presumably experience many of the same confounding factors but have been breastfed for different lengths of time $(28,29)$. These studies have had mixed results with one suggesting that residual confounding explains the relationship (28) and the other suggesting that breastfeeding itself may provide modest protection against obesity (29). While this study design provides additional information on the effects of confounding factors, it does not adequately account for within-child confounders, such as temperament or health problems that may explain why siblings were not breastfed for the same duration. Last, randomized interventions that improve breastfeeding duration rates have been used to remove some of the effects of confounding factors. For example, the PROBIT trial did not find any differences in growth outcomes in 6-year-old children as a function of duration of breastfeeding (30). While this approach clearly has benefits in removing some bias, the intervention itself may cause additional bias. For example, the PROBIT trial intervention included instructions in parenting practices, such as feeding the baby "on demand," which may selectively encourage caregivers in the intervention group to engage in more baby-led feeding practices. Clearly, no study design is capable of removing all bias except a truly randomized intervention assigning mother-baby dyads to either breast- or formula feeding, which is an unrealistic and unethical research design.

In sum, confounding factors may play a significant role in explaining the association between breastfeeding and lower risk of overweight later in life. The currently available literature demonstrates mixed and inconclusive results, in part because no study design can remove every form of bias. The subsequent sections of the paper outline differences in the feeding experience that are directly related to feeding mode and how these differences can impact the 
infant's short- and long-term risk for overweight and obesity.

\section{Behavioral factors}

From a lifespan perspective, feeding and eating during infancy are unique because the infant is almost entirely dependent on his or her caregiver for the attainment and delivery of food. Thus, although the infant is an active agent during the feeding interaction, the caregiver has the potential to exert control over what, when, and even how much the infant consumes (31). As evidenced by the research reviewed below, this unique situation, and the nature of interactions that occur between caregivers and infants during this situation, have implications for the infant's net energy balance and his/her developing abilities to regulate energy intake.

Several lines of research demonstrate that infants have at least some ability to appropriately regulate intake to meet nutritional needs. At a very young age, both breast- and formula-fed infants exhibit an ability to regulate meal size and interval in response to and in anticipation of individual feeding and sleeping schedules (32-34). Infants also appear to be responsive to alterations in energy density (caloric content) and supply of breast milk and formula when they are allowed to self-direct feeding behavior. For example, Fomon and colleagues have shown that infants decrease the volume of formula consumed when the energy density is increased, resulting in consumption of approximately the same number of calories per day $(35,36)$. Dewey and colleagues have shown that, in breastfed infants, daily breast milk intake is inversely associated with both fat content of the milk and its energy density (37), and some breastfed infants are capable of regulating intake even when the breasts are stimulated to over-produce (38).

However, it is important to note that this selfregulation of intake may take several weeks to appear and may not be tightly regulated in all infants. In Fomon's study of caloric density of formulas, infants fed formula of a higher caloric density consumed significantly more energy and gained significantly more weight for the first six weeks of the manipulation than infants fed a standard formula. After six weeks, energy intake was similar between the high and normal caloric density formula groups $(35,36)$. In Dewey and colleagues' study of induced milk overproduction, all infants significantly increased intake and consumed more breast milk immediately after the manipulation, but half of infants eventually adjusted intakes back to baseline levels while the other half of breastfed infants' intakes remained elevated in response to increased milk supply (38). Thus, although infants do appear to have some intake-regulation ability early in life, the above evidence illustrates: (1) infants do initially respond to characteristics of breast milk and/or formula (e.g., energy density, supply) as well as to characteristics of the environment (e.g., feeding and sleeping schedule), and (2) not all infants are able to eventually readjust intake back to baseline levels after caregiver interventions. Individual differences in the ability to self-regulate intake may be due to genetic differences in metabolism and/or appetite regulating pathways. In addition, a significant body of literature suggests that differences in the prenatal and perinatal environment can "program" an infant's metabolome, appetite regulation pathways, energy balance, and metabolism, leading to differences in nutritional intake, growth, and adiposity that have potential life-long implications for risk of obesity and obesity-related chronic diseases (39-41).

Evidence suggests that individual differences in responsiveness to dietary characteristics and energy balance may be partially attributable to caregiver behaviors, which can potentially override intake regulation abilities when bottle-feeding or when feeding solid foods. During bottle-feeding, mothers can visually assess and monitor how much infants are consuming, and can encourage over-consumption by the infant. In contrast, the act of breastfeeding does not provide this key visual information about how much milk is consumed. Thus, breastfeeding mothers lack the ability to directly assess and monitor intake and must rely on other information (e.g., satiety cues from the infant) to determine feeding adequacy. For this reason, it is hypothesized that breastfeeding mothers are more likely to trust the infant's ability to self-regulate and be attentive to the infant's expressions and cues indicating fullness and satiation (31).

Observational studies of Caucasian mother-infant dyads from Western cultures provide evidence that this hypothesis may be true. Wright and colleagues observed that, shortly after birth, both breastfed and bottle-fed infants displayed reasonably regular and consistent intake patterns (33). However, at two months of age breastfed infants exhibited a diurnal pattern, taking a larger feed in the morning and smaller feeds throughout the remainder of the day while bottle-fed infants still consumed feeds of equal size at all points during the day. The more consistent timing and volume of feeds in bottle-fed infants suggests that parental control, rather than infant self-regulation, was driving intake patterns (33). In addition, other researchers have observed that during the first few months after birth, bottle-feeding mothers are significantly more likely to start, end, interrupt and determine the outcome of most feeding sessions, whereas breastfeeding dyads are characterized by more equal division between infant 
and mother for control over the progress and outcome of the feed $(33,42,43)$.

One study has provided clear evidence that caregiver behaviors can have a direct influence on infant intake and growth. In a study of 84 formulafed infants with weighed dietary intake records, mothers were classified on the basis of how frequently they "emptied the bottle" ( $<10 \mathrm{~mL}$ remaining in the bottle) and whether large $(>6 \mathrm{oz})$ or small ( $\leq 6 \mathrm{oz})$ servings of formula were prepared at 3 months of age. Infants of mothers who routinely ( $>50 \%$ of feeds) emptied the bottle had greater body fatness at five months of age $(44,45)$. Additionally, mothers who reported dispensing large servings of formula at feedings had infants with significantly greater formula intake at three months and greater weight gain between three and five months. While it may be that infant intake was driving these feeding behaviors, no differences in intake or weight differences were seen at one month of age.

Studies also suggest that the patterns of caregiver control over feeding established during infancy may extend later into childhood. Heinig and colleagues reported that breastfed infants given solid foods between three and six months of age compensated for the additional calories by decreasing breast milk intakes to the extent that the total amount of calories consumed did not differ from their exclusively breastfed counterparts (46). In contrast, the formula intake of bottle-fed infants did not decline, likely because caregivers had expectations about the typical volume of formula their infants consumed at feedings and continued to promote this intake without adjusting for the addition of solid foods to the diet. Additionally, there is evidence that the trust breastfeeding mothers learn from early feeding experience may translate into less controlling feeding practices later in childhood. Taveras and colleagues found that mothers who breastfed for longer durations were less likely to restrict their child's dietary intake at 1 year (47). Additionally, Farrow and Blisset reported that mothers who breastfed for longer durations used less control over child feeding and were more sensitive to child cues at mealtimes, which predicted more positive mother-child mealtime interactions at 1 year (48). In a slightly older sample, Fisher and colleagues found that mothers who had breastfed their infant for at least 12 months used less control over feeding and had leaner infants at 18 months (49). Caregivers who are more controlling of their preschool-aged children's intakes have children with poorer self-regulation of dietary intake and higher adiposity (50), thus, the association between breastfeeding and lowered obesity risk may be related to long-term feeding practices that develop through experience with either breast or bottle-feeding.

In summary, the evidence available to date suggests that infants' emerging ability to self-regulate intake can be overridden by caregiver behaviors, and that the caregiver feeding experience of breastfeeding and formula feeding may promote the development of long-term differences in parental control of child food intake. These studies provide direct and indirect evidence that parental control of infant food intake has the potential to lead to infant intakes that exceed energy needs for appropriate growth. To date, most studies are observational, which limits establishment of causation or directionality. For example, breastfeeding may promote less maternal control of food intake, but mothers also may choose to breastfeed if this control is not as important to them. Additionally, if a formula-fed infant is heavier at the end of one year, a mother's use of control over feeding may develop in response to her child's current weight status at one year, and not necessarily as a result of previous experiences.

\section{Physiological factors}

Human breast milk contains hundreds of components, many of which have the potential to affect short- and long-term growth patterns of children $(51,52)$. Inter-species variability in milk composition is considerable $(51,53)$ and may in part explain the quantity and quality of growth experienced prior to weaning $(52,54)$. Early human growth is characterized by relatively slow growth in physical size (length, weight) but substantial growth in brain volume (55). Thus, human milk contains proportionally more lactose for fueling the metabolism of the central nervous system, and specific fats and cholesterol for building central nervous system tissues $(51,53)$. In contrast, protein and mineral content is relatively higher in the milk consumed by animals that experience substantial and rapid gains in physical size, such as the cow, which doubles its birth weight in just 47 days $(52,54)$. Thus, the higher protein and mineral content of cow's milk supports early, rapid development of skeletal and smooth muscle, bone, and connective tissues $(52,54)$. As much of today's formula is manufactured from cow's milk, formula's macro- and micro-nutrient composition still contain key differences from human breast milk.

Experimental evidence supports the assertion that the protein content of some formulas may promote excess physical growth in infants. Early studies were focused on comparing breastfed infants and formula-fed infants, as formula typically contains double the protein of breast milk on a per-kilocalorie basis (56). The results of these early studies were 
equivocal (56-58), largely because researchers did not carefully control for factors, such as lifestyle, timing of solid foods, or duration and exclusivity of breastfeeding practices. A more recent study that carefully controlled these factors, has shown that weight-for-length and body mass index (BMI) are higher at 12 and 24 months in children receiving formula with $3 \mathrm{~g}$ protein $/ 100 \mathrm{kcal}$ as compared with children receiving both lower $(1.8 \mathrm{~g}$ protein/ $100 \mathrm{kcal}$ ) protein formula and breast milk (59). As all infants consumed the same volume of milk or formula, the results suggest that protein content may exert influences on growth independently of caloric intake.

In addition, differences in fatty acid profile of breast milk versus formula may contribute to differential risk of obesity. Recent research in animals suggests that the omega-6/omega- 3 ratio found in formulas may stimulate adipocyte growth and differentiation $(60,61)$. In addition, the omega-6/omega-3 ratio found in formula may promote more inflammation in the infant's body. A substantial body of research demonstrates the role of inflammation in the progression of obesity-related diseases, such as diabetes, cardiovascular disease, and cancer, and recent research supports an independent role of inflammation in the development of obesity early in life (62). The role of breast milk omega- 3 fatty acids and other factors in decreasing inflammation may reduce the risk of later obesity by acting on regulators of food intake found within the central nervous system, as well as peripherally in the regulation of metabolism (62).

Breast milk also contains numerous bioactive factors that have the potential to regulate growth in humans, such as immunoglobulins, live cells, enzymes, pituitary hormones, steroid hormones, cytokines, chemokines, brain-gut peptides, growth factors, and various nutritional constituents (proteins, lipids, carbohydrates) functioning in nonnutritive roles $(63,64)$. The role of these bioactive components of breast milk and regulation of growth is a relatively new field of study. Recent research has focused on simply confirming the presence of growth-regulating components, such as leptin, ghrelin, insulin-like growth factor-1, resistin, and adiponectin in human milk; comparing serum levels of bioactive components in breastfed and formula-fed infants; and relating serum levels to infant growth and/or maternal weight status (65-69). While little is known about whether these bioactive milk components regulate the growth of infants, this has become an active area of research given their effects on appetite regulation in adults (70).

Leptin acts as a satiety factor, a regulator of energy expenditure, and regulator of many neuroendocrine axes (20). Research has documented its presence in breast milk and absence in formula $(71,72)$. Additionally, most studies have documented that formula-fed infants have significantly lower blood leptin levels than breastfed infants $(65,68)$. Subsequent research has shown that milk leptin levels are related to maternal plasma leptin concentration and maternal body mass index and that milk leptin levels are correlated to infant serum leptin concentration (68). Given that maternal milk leptin levels are negatively associated with infant weight gain across early childhood $(73,74)$, breast milk leptin may contribute to appetite and growth in infants. While leptin is not likely to be the only solution to the obesity crisis (75), preliminary research in animals (76) is promising enough that supplementation of formula with leptin (77) and leptin infusions for infants (78) have been proposed. However, caution should be used when interpreting this research as the fat in breast milk artificially elevates radioimmuoassy-measured leptin levels (79). When defatted human milk samples are analyzed, the leptin content is significantly lower and may not be enough to affect circulating levels in the infant (79).

In summary, macronutrient profiles and bioactive factors differ between breast milk and formula and this difference may result in long-term growth disparities in infants. The increased protein content and ratio of omega-6/omega-3 fatty acids found in formulas may promote greater physical growth in formula-fed infants. Additionally, a variety of bioactive factors in human milk may regulate infant metabolism, appetite, and caloric intake, producing wide-ranging physiological effects on body fat levels and weight gain patterns.

\section{Early growth differences as a risk factor}

Research completed during the last two decades supports the assertion that the quantity and quality of growth during the first years of life differs between breastfed and formula-fed infants. In a review article summarizing the results of nineteen well-controlled studies comparing growth of breastfed and formulafed infants, Dewey demonstrates that almost all studies have shown significant differences in weight or weight gain patterns by feeding mode (80). Infants who are breastfed for nine months are, on average, $400 \mathrm{~g}$ lighter than formula-fed infants by the end of the first year; after 12 months of breastfeeding this difference increases to $600-650 \mathrm{~g}$ (80). In addition, excess weight gain in formula-fed infants does not appear to be offset by higher gains in length. More recent research has suggested that the period from 3 to 6 months is when feeding mode 
may exhibit the greatest effect on growth patterns (81).

A natural question that follows is, "what is the composition of the extra weight gain?" Due to the invasiveness, expertise, time, and equipment necessary for the study of body composition during infancy, few researchers have examined whether formula-fed and breastfed infants exhibit differing body fatness levels. A few early studies using skinfold measurements have reported conflicting results (80). A subsequent study using more technologically advanced methods did not show consistent effects of feeding mode on body fatness (82). However, close inspection of the feeding practices of the breastfeeding group showed early cessation of breastfeeding and significant formula use. Not surprisingly, the two groups did not differ in weight or weight velocity except at a few select time points. When feeding mode and lifestyle factors are carefully controlled, body fatness is significantly higher in formula-fed infants from the second 6 months of life until at least 24 months of age (83).

While "chubby" babies have historically been viewed as healthy babies, new research suggests that the pattern of excess weight and fat gains early in life seen with formula feeding may be a risk factor for both overweight and obesity-related diseases later in life. An emerging area of study is rapid weight gain, defined as upward crossing through at least one centile band on US and European growth charts during the first 1-2 years of life, and risk of later obesity. Recent review and meta-analysis papers report that nearly all studies done to date demonstrate that rapid weight gain increases the risk of obesity later in life (84-86). The risk of later obesity is on average two- to three-fold higher for infants with rapid weight gain (86), with the population attributable risk of obesity during young adulthood as a result of rapid weight gain during infancy estimated to be $30 \%$ (87).

While rapid weight gain may be a risk factor for later overweight, not all children who grow rapidly during infancy become overweight later in life. In a study of German children, Toschke et al. have shown that the odds ratio for risk of overweight in early childhood (aged 5-7 years) is 5.7 if the child experienced rapid weight gain (>9 $764 \mathrm{~g}$ ) from birth to age two (88). However, the positive predictive value of this definition of rapid weight gain was only $19 \%$, meaning that only 1 in 5 children experiencing rapid weight gain during childhood actually became overweight later in childhood. Understanding which children experiencing rapid growth are at the highest risk for later obesity will be a critical part of future research so that interventions can be targeted to children most at risk (86). Whether the early feeding experience is a critical factor in the differential outcomes of children with rapid weight gain is unknown.

\section{Conclusions and future directions}

The research outlined above supports the hypothesis that the negative association between breastfeeding and later overweight is likely in part due to confounding lifestyle factors, but also may be due to infant and maternal behavioral differences between breastfeeding and bottle-feeding, as well as physiological differences in the infant that occur based on the differing composition of breast milk and formula. These behavioral and physiological differences may contribute to subtle changes in the infant's metabolome that affect appetite, food intake, and metabolism, as well as more overtly affect energy intake and net energy balance.

The evidence we have reviewed suggests that infants appear to have an emerging ability to adjust energy intake in response to characteristics of breast milk and/or formula (e.g., energy density), as well as to characteristics of the environment (e.g., day vs. night, sleeping schedule). Due to natural limitations posed by the breastfeeding process, caregivers have a limited ability to manipulate the intake of the breastfed infant. Theoretically, this would provide the infant with opportunities to develop self-regulation capabilities and maintain energy balance in response to dietary characteristics, growth, or activity levels. In contrast, bottle-feeding caregivers can interfere with this emerging ability by taking control of initiating or terminating the feed, encouraging infants to "finish the bottle," dispensing too much formula at a feed, and engaging in controlling or restrictive feeding practices when their children are older. Infants appear to have varying capabilities to respond to dietary characteristics. Whether this is due to variation in underlying genetic traits; differences in the infant's metabolome that regulate appetite, food intake, or energy expenditure; or simply a failure of the study design to account for caregiver interference with infant regulation is unknown. Studies investigating the effect of prenatal and neonatal experiences (programming) will hopefully shed light on this issue in the future. The constituents of breast milk also may provide protection against later obesity, as the nutrient profile is optimally balanced to promote the quality and quantity of growth that is appropriate for our species. In addition, growth-regulating components within the milk, such as leptin, ghrelin, adiponectin, and insulin, may directly influence energy intake, energy expenditure, growth, and body composition. Breastfed infants weigh less and are leaner throughout the early years of life, which may in part be due 
to these behavioral and physiological differences in the early feeding experience.

While we reviewed behavioral and physiological difference separately, they are part of a dynamic, interrelated system affecting infant growth and development. For example, infant and caregiver behaviors are likely to not be discrete in early infancy; infant behaviors may be a reaction to caregiver behaviors and characteristics, just as caregiver interactions may be in response to infant behaviors and characteristics. Additionally, research suggests that the infant and breast provide reciprocal feedback and regulation capabilities. Historically, breasts have been viewed as a simplistic "supply and demand system," where infant intake drives milk production (89). More in depth examination of intake of infants and milk storage capacity of breasts suggests that infant intake can be limited by the breasts, such as when one breast is less productive than the other or when the breast's milk storage is at a temporarily lower point (90). The complexity of this system presents many challenges and opportunities for future research in this field. Future research should apply interdisciplinary approaches to better understand the interactions of these components, rather than simply focusing on one element within the system.

Not all breastfed infants become lean and not all formula-fed infants become obese; and not all overweight children become overweight adults. A better understanding of the interactions among the components that comprise the infant feeding experience may provide insights into why this is so. As today's breastfeeding families are likely to engage in supplemental bottle-feeding of breast milk and/or formula (91), this review of the mechanisms underlying the association between infant feeding experience and later overweight and obesity supports the assertion that all families may benefit from prevention programs or education that encourage: 1) exclusivity and duration of breastfeeding as outlined in World Health Organization guidelines (92), 2) healthy lifestyle practices in the family before and after the birth of the child, 3) child-led rather than parent-led feeding practices and behaviors, and 4) use of donor (banked) human milk whenever supplemental nutrition is needed. A clearer understanding of which aspects of the infant feeding experience have causal influence on the development of infant eating behaviors and weight status will provide further insight into how to effectively foster healthy growth and development in all infants, regardless of what and how they are initially fed.

Declaration of interest: The authors report no conflicts of interest. The authors alone are responsible for the content and writing of the paper.

\section{References}

1. James WP. The challenge of childhood obesity. Int J Pediatr Obes. 2006;1:7-10.

2. Wang Y, Lobstein T. Worldwide trends in childhood overweight and obesity. Int J Pediatr Obes. 2006;1:11-25.

3. Whitlock EP, Williams SB, Gold R, et al. Screening and Interventions for Childhood Overweight: A Summary of Evidence for the US Preventive Services Task Force. Pediatrics. 2005;116:e125-44.

4. US Preventive Services Task Force. Screening and Interventions for Overweight in Children and Adolescents: Recommendation Statement. Pediatrics. 2005;116:205-9.

5. Koplan JP, Liverman CT, Kraak VI. Preventing childhood obesity: health in the balance: executive summary. J Am Diet Assoc. 2005;105:131-8.

6. Krebs NF, Jacobson MS. American Academy of Pediatrics Committee on Nutrition. Prevention of Pediatric Overweight and Obesity. Pediatrics. 2003;112:424-30.

7. Daniels SR, Arnett DK, Eckel RH, et al. Overweight in children and adolescents: pathophysiology, consequences, prevention, and treatment. Circulation. 2005;111:1999-2012.

8. Kramer MS. Do breast-feeding and delayed introduction of solid foods protect against subsequent obesity? J Pediatr. 1981;98:883-7.

9. Armstrong J, Reilly JJ, Team TCHI. Breastfeeding and lowering the risk of childhood obesity. Lancet. 2002;359: 2003-4.

10. Gillman MW, Rifas-Shiman SL, Camargo CA Jr, et al. Risk of overweight among adolescents who were breastfed as infants. JAMA. 2001;285:2461-7.

11. Victora CG, Barros F, Lima RC, et al. Anthropometry and body composition of 18 year old men according to duration of breast feeding: birth cohort study from Brazil. BMJ. 2003; 327:901.

12. Bogen DL, Hanusa BH, Whitaker RC. The effect of breastfeeding with and without concurrent formula feeding on the risk of obesity at 4 years of age: a retrospective cohort study. Obes Res. 2004;12:1527-35.

13. von Kries R, Koletzko B, Sauerwald T, et al. Breast feeding and obesity: cross sectional study. BMJ. 1999;319:147-50.

14. Dewey KG. Is breastfeeding protective against child obesity? J Hum Lact. 2003;19:9-18.

15. Arenz S, Ruckerl R, Koletzko B, et al. Breast-feeding and childhood obesity-a systematic review. Int J Obes Relat Metab Disord. 2004;28:1247-56.

16. Owen CG, Martin RM, Whincup PH, et al. Effect of infant feeding on the risk of obesity across the life course: a quantitative review of published evidence. Pediatrics. 2005; 115:1367-77.

17. Harder T, Bergmann R, Kallischnigg G, et al. Duration of breastfeeding and risk of overweight: a meta-analysis. Am J Epidemiol. 2005;162:397-403.

18. Collins LM, Murphy SA, Nair VN, et al. A strategy for optimizing and evaluating behavioral interventions. Ann Behav Med. 2005;30:65-73.

19. Greenberg MT. Current and future challenges in schoolbased prevention: the researcher perspective. Prev Sci. 2004; 5:5-13.

20. Agostoni C. Ghrelin, leptin and the neurometabolic axis of breastfed and formula-fed infants. Acta Paediatr. 2005;94: 523-5.

21. Hendricks K, Briefel R, Novak T, et al. Maternal and child characteristics associated with infant and toddler feeding practices. J Am Diet Assoc. 2006;106:S135-48.

22. Dietz W. Factors associated with childhood obesity. Nutrition. 1991;7:290-1. 
23. Moore DB, Howell PB, Treiber FA. Changes in overweight in youth over a period of 7 years: impact of ethnicity, gender and socioeconomic status. Ethn Dis. 2002;12(S1):83-6.

24. Francis LA, Ventura AK, Marini M, et al. Parent overweight predicts daughters' increase in BMI and disinhibited overeating from 5 to 13 years. Obesity (Silver Spring). 2007;15: 1544-53.

25. Hilson JA, Rasmussen KM, Kjolhede CL. High prepregnant body mass index is associated with poor lactation outcomes among white, rural women independent of psychosocial and demographic correlates. J Hum Lact. 2004;20:18-29.

26. Rasmussen KM, Hilson JA, Kjolhede CL. Obesity as a risk factor for failure to initiate and sustain lactation. Adv Exp Med Biol. 2002;503:217-22.

27. Wadsworth M, Marshall S, Hardy R, et al. Breast feeding and obesity. Relation may be accounted for by social factors. BMJ. 1999;319:1576.

28. Nelson MC, Gordon-Larsen P, Adair LS. Are adolescents who were breast-fed less likely to be overweight? Analyses of sibling pairs to reduce confounding. Epidemiology. 2005;16: 247-53.

29. Gillman MW, Rifas-Shiman SL, Berkey CS, et al. Breastfeeding and overweight in adolescence: within-family analysis [corrected]. Epidemiology. 2006;17:112-4.

30. Kramer MS, Matush L, Vanilovich I, et al. Effects of prolonged and exclusive breastfeeding on child height, weight, adiposity, and blood pressure at age $6.5 \mathrm{y}$ : evidence from a large randomized trial. Am J Clin Nutr. 2007;86: 1717-21.

31. Wright P. Hunger, Satiety and Feeding Behavior in Early Infancy. In: Boakes R, Popplewell D, Burton M, (eds). Eating Habits: Food, Physiology and Learned Behavior. New York: John Wiley and Sons, 1987. p. 75-95.

32. Matheny RJ, Birch LL, Picciano MF. Control of intake by human-milk-fed infants: relationships between feeding size and interval. Dev Psychobiol. 1990;23:511-8.

33. Wright P, Fawcett J, Crow R. The development of differences in the feeding behaviour of bottle and breast fed human infants from birth to two months. Behav Process. 1980;5:1-20.

34. Wright P. Development of feeding behaviour in early infancy: implications for obesity. Health Bull (Edinb). 1981;39: 197-205.

35. Fomon SJ, Filer LJ Jr, Thomas LN, et al. Relationship between formula concentration and rate of growth of normal infants. J Nutr. 1969;98:241-54.

36. Fomon SJ, Filmer LJ Jr, Thomas LN, et al. Influence of formula concentration on caloric intake and growth of normal infants. Acta Paediatr Scand. 1975;64:172-81.

37. Dewey KG, Heinig MJ, Nommsen LA, et al. Maternal versus infant factors related to breast milk intake and residual milk volume: the DARLING study. Pediatrics. 1991;87:829-37.

38. Dewey KG, Lonnerdal B. Infant self-regulation of breast milk intake. Acta Paediatr Scand. 1986;75:893-8.

39. Muhlhausler BS. Programming of the appetite-regulating neural network: a link between maternal overnutrition and the programming of obesity? J Neuroendocrinol. 2007;19: $67-72$.

40. Cottrell EC, Ozanne SE. Developmental programming of energy balance and the metabolic syndrome. Proc Nutr Soc. 2007;66:198-206.

41. Cripps RL, Martin-Gronert MS, Ozanne SE. Fetal and perinatal programming of appetite. Clin Sci (Lond). 2005; 109:1-11.

42. Dunn J. Feeding and sleeping. In: Rutter M, ed. Scientific foundations of developmental psychiatry. London: Heinemann Medical Books, 1980. p. 119-28.
43. Crow RA, Fawcett JN, Wright P. Maternal behavior during breast- and bottle-feeding. J Behav Med. 1980;3:259-77.

44. Dewey KG, Nommsen-Rivers LA, Lonnerdal B. Plasma insulin and insulin-releasing amino acid (IRAA) concentrations are higher in formula fed than breastfed infants at 5 months of age. Experimental Biology. 2004; abstract \#1124.

45. Dewey KG. Breastfeeding and other infant feeding practices that may influence child obesity. In: Birch LL, Dietz W, (eds). Eating behaviors of the young child: prenatal and postnatal influences on healthy eating. Elk Grove Village, IL: American Academy of Pediatrics, 2008. p. 69-93.

46. Heinig MJ, Nommsen LA, Peerson JM, et al. Intake and growth of breast-fed and formula-fed infants in relation to the timing of introduction of complementary foods: the DARLING study. Davis Area Research on Lactation, Infant Nutrition and Growth. Acta Paediatr. 1993;82:999-1006.

47. Taveras EM, Scanlon KS, Birch L, et al. Association of breastfeeding with maternal control of infant feeding at age 1 year. Pediatrics. 2004;114:e577-83.

48. Farrow C, Blissett J. Breast-feeding, maternal feeding practices and mealtime negativity at one year. Appetite. 2006;46: 49-56.

49. Fisher JO, Birch LL, Smiciklas-Wright $\mathrm{H}$, et al. Breastfeeding through the first year predicts maternal control in feeding and subsequent toddler energy intakes. J Am Diet Assoc. 2000;100:641-6.

50. Birch LL, Fisher JO. Development of eating behaviors among children and adolescents. Pediatrics. 1998;101:539-48.

51. Jensen RG, ed. Handbook of Milk Composition. San Diego: Academic Press, 1995.

52. Hambraeus L. Proprietary milk versus human breast milk in infant feeding. A critical appraisal from the nutritional point of view. Pediatr Clin North Am. 1977;24:17-36.

53. Jensen RG. Introduction. In: Jensen RG, ed. Handbook of Milk Composition. San Diego: Academic Press, 1995. p. 1-3.

54. Oftedal OT, Iverson SJ. Comparative analysis of nonhuman milks: phylogenetic variation in the gross composition of milks. In: Jensen RG, ed. Handbook of Milk Composition. San Diego: Academic Press, 1995. p. 749-89.

55. Legarraga $\mathrm{H}$. Growth in infancy and childhood: a pediatric approach. In: Cameron N, ed. Human growth and development. Amsterdam: Academic Press, 2006. p. 21-44.

56. Raiha NCR, Nesci AF, Cajozzo $C$ et al. Protein quantity and quality in infant formula: closer to the reference. In: Raiha NCR, Rubaltelli FF, (eds). Infant formula: closer to the reference. Philadelphia: Levey/Lippincott Williams \& Wilkins, 2002. p. 111-20.

57. Ziegler EE. Protein requirements in infancy. In: Raiha NCR, Rubaltelli FF, (eds). Infant formula: closer to the reference. Philadelphia: Levey/Lippincott Williams \& Wilkins, 2002. p. $97-110$.

58. Heinig MJ, Nommsen LA, Peerson JM, et al. Energy and protein intakes of breast-fed and formula-fed infants during the first year of life and their association with growth velocity: the DARLING Study. Am J Clin Nutr. 1993;58:152-61.

59. EU Childhood Obesity Programme. EU Childhood Obesity Programme press pack. Budapest; 2007. April 20, 2007.

60. Ailhaud G, Guesnet P. Fatty acid composition of fats is an early determinant of childhood obesity: a short review and an opinion. Obes Rev. 2004;5:21-6.

61. Ailhaud G, Massiera F, Weill P, et al. Temporal changes in dietary fats: role of $n-6$ polyunsaturated fatty acids in excessive adipose tissue development and relationship to obesity. Prog Lipid Res. 2006;45:203-36.

62. Das UN. Is obesity an inflammatory condition? Nutrition. 2001;17:953-66. 
63. Read LC, Penttila IA, Howarth GS, et al. Role and function of growth factors in infant nutrition. In: Raiha NCR, Rubaltelli FF, (eds). Infant formula: closer to the reference. Philadelphia: Vevey/Lippincott Williams \& Wilkins, 2002. p. 185-95.

64. Hamosh M. Bioactive factors in human milk. Pediatr Clin North Am. 2001;48:69-86.

65. Savino F, Fissore MF, Grassino EC, et al. Ghrelin, leptin and IGF-I levels in breast-fed and formula-fed infants in the first years of life. Acta Paediatr. 2005;94:531-7.

66. Elmlinger MW, Hochhaus F, Loui A, et al. Insulin-like growth factors and binding proteins in early milk from mothers of preterm and term infants. Horm Res. 2007;68: 124-31.

67. Martin LJ, Woo JG, Geraghty SR, et al. Adiponectin is present in human milk and is associated with maternal factors. Am J Clin Nutr. 2006;83:1106-11.

68. Savino F, Liguori SA. Update on breast milk hormones: leptin, ghrelin and adiponectin. Clin Nutr. 2008;27:42-7.

69. Aydin S, Ozkan Y, Erman F, et al. Presence of obestatin in breast milk: relationship among obestatin, ghrelin, and leptin in lactating women. Nutrition. 2008;24:689-93.

70. Valassi E, Scacchi M, Cavagnini F. Neuroendocrine control of food intake. Nutr Metab Cardiovasc Dis. 2008;18:158-68.

71. O'Connor D, Funanage V, Locke R, et al. Leptin is not present in infant formulas. J Endocrinol Invest. 2003;26:490.

72. Resto M, O'Connor D, Leef $\mathrm{K}$, et al. Leptin levels in preterm human breast milk and infant formula. Pediatrics. 2001;108: E15.

73. Dundar NO, Anal O, Dundar B, et al. Longitudinal investigation of the relationship between breast milk leptin levels and growth in breast-fed infants. J Pediatr Endocrinol Metab. 2005;18:181-7.

74. Miralles O, Sanchez J, Palou A, et al. A physiological role of breast milk leptin in body weight control in developing infants. Obesity (Silver Spring). 2006;14:1371-7.

75. Uysal FK, Onal EE, Aral YZ, et al. Breast milk leptin: its relationship to maternal and infant adiposity. Clin Nutr. 2002;21:157-60

76. Pico C, Oliver P, Sanchez J, et al. The intake of physiological doses of leptin during lactation in rats prevents obesity in later life. Int J Obes (Lond). 2007;31:1199-209.

77. Paul AM. Baby's first diet pill. New York Times. August 5, 2007.
78. Cole TJ. Early causes of child obesity and implications for prevention. Acta Paediatr Suppl. 2007;96:2-4.

79. Lonnerdal B, Havel PJ. Serum leptin concentrations in infants: effects of diet, sex, and adiposity. Am J Clin Nutr. 2000;72:484-9.

80. Dewey KG. Growth characteristics of breast-fed compared to formula-fed infants. Biol Neonate. 1998;74:94-105.

81. Kramer MS, Guo T, Platt RW, et al. Feeding effects on growth during infancy. J Pediatr. 2004;145:600-5.

82. Butte NF, Wong WW, Hopkinson JM, et al. Infant feeding mode affects early growth and body composition. Pediatrics. 2000;106:1355-66.

83. Dewey KG, Heinig MJ, Nommsen LA, et al. Breast-fed infants are leaner than formula-fed infants at $1 \mathrm{y}$ of age: the DARLING study. Am J Clin Nutr. 1993;57:140-5.

84. Baird J, Fisher D, Lucas P, et al. Being big or growing fast: systematic review of size and growth in infancy and later obesity. BMJ. 2005;331:929.

85. Monteiro PO, Victora CG. Rapid growth in infancy and childhood and obesity in later life-a systematic review. Obes Rev. 2005;6:143-54.

86. Ong KK, Loos RJ. Rapid infancy weight gain and subsequent obesity: systematic reviews and hopeful suggestions. Acta Paediatr. 2006;95:904-8.

87. Stettler N, Kumanyika SK, Katz SH, et al. Rapid weight gain during infancy and obesity in young adulthood in a cohort of African Americans. Am J Clin Nutr. 2003;77:1374-8.

88. Toschke AM, Grote V, Koletzko B, et al. Identifying children at high risk for overweight at school entry by weight gain during the first 2 years. Arch Pediatr Adolesc Med. 2004;158: 449-52.

89. Lawrence RA, Lawrence RM. Physiology of breastfeeding. Breastfeeding: A guide for the medical profession. Philadelphia: Elsevier Mosby, 2005. p. 105-70.

90. Kent JC, Mitoulas LR, Cregan MD, et al. Volume and frequency of breastfeedings and fat content of breast milk throughout the day. Pediatrics. 2006;117:e387-95.

91. Li R, Zhao Z, Mokdad A, et al. Prevalence of breastfeeding in the United States: the 2001 National Immunization Survey. Pediatrics. 2003;111:1198-201.

92. The optimal duration of exclusive breastfeeding: Report of an expert consultation. WHO/NHD/01.09, WHO/FCH/CAH/ 01.24; Geneva: World Health Organization, 2001. 